\title{
Progression of vinyl chloride induced hepatic fibrosis to angiosarcoma of the liver
}

\author{
D B JONES AND P M SMITH
}

From the Department of Gastroenterology, Llandough Hospital, Penarth, S Glamorgan, UK

ABSTRACT Two vinyl chloride monomer (VCM) workers, who developed non-cirrhotic portal fibrosis and portal hypertension, died from angiosarcoma of the liver five and ten years later respectively, despite withdrawal from occupational exposure. We suggest that non-cirrhotic portal fibrosis caused by exposure to VCM is potentially premalignant and that those workers who already have the condition should be carefully monitored.

Since the original communication by Creech and Johnson' there have been several further case reports of vinyl chloride monomer (VCM) induced angiosarcoma of the liver. A commoner hepatic lesion is non-cirrhotic portal fibrosis ${ }^{2}$ leading to portal hypertension. It has been suggested that this lesion may be a precursor to angiosarcoma formation, ${ }^{3-5}$ but there has been only one report of a patient with documented hepatic fibrosis developing angiosarcoma at a later date. ${ }^{\circ}$ We describe two further cases.

\section{Case reports}

CASE 1

A 60 -year old white man initially presented in 1969 to the dermatology department with a skin eruption. Examination at that time showed anaemia, thrombocytopenia, hepatosplenomegaly, and occult faecal blood loss. He had an occupational history of exposure to VCM at high concentration for seven years while working as a polycleaner and a blowdown recovery operator. After two haematemeses, barium studies and splenic venography confirmed portal hypertension and oesophageal varices, and he underwent an end-to-side portocaval shunt. At laparotomy the liver looked nodular, but operative liver biopsy showed non-cirrhotic portal fibrosis only. Over the next four years the patient developed chronic portosystemic encephalopathy and maturity onset diabetes mellitus that was treated with oral hypoglycaemic agents. In May 1980 he presented with worsening mental deterioration, hepatic foetor, asterixis, and an enlarging liver. Liver function test

Received 5 October 1981

Accepted 20 November 1981 results showed a normal serum bilirubin with a raised alkaline phosphatase (201 IU/1) and $\gamma$-glutamyl transpeptidase (83 IU/1). Radioisotope liver scan showed a small liver with no filling defects and needle liver biopsy showed a well-pronounced micronodular cirrhosis with no evidence to tumour. $\mathrm{He}$ responded to protein restriction and lactulose and was discharged. Within a week of discharge he was readmitted with an endoscopically confirmed bleeding duodenal ulcer. After this, he lapsed into hepatic coma and died. Necropsy showed multiple malignant tumours in the liver (wt $1130 \mathrm{~g}$ ), one of which was haemorrhagic. Histologically, a great variety of liver cell lesions were present, some resembling angiosarcomas, some hepatocarcinomas, and some adenomas. There were also atypical sinusoidal cells and fibrosis in the non-tumorous parts of the liver.

\section{CASE 2}

A 49-year-old man with a 12-year history of exposure to vinyl chloride monomer while working as a spray drier bagger, premix operator, and paste charging operator was, during a factory survey of process workers in 1974, found to have thrombocytopenia. This was later shown to be due to hypersplenism and presinusoidal portal hypertension. He was otherwise well and drank five pints of beer a night. Liver function test results were normal apart from a raised $\gamma$-glutamyl transpeptidase level of $82 \mathrm{IU} / 1$. Varices were shown by barium studies and endoscopy, and liver biopsy showed fatty change and slight non-cirrhotic portal fibrosis. Two years later the patient developed insulin dependent diabetes mellitus, but otherwise remained well until October 1979 when he presented with a large haematemesis. Endoscopy confirmed oesophageal 306 
varices to be the source of haemorrhage, and these were treated by injection sclerotherapy. The serum alkaline phosphatase and $\gamma$-glutamyl transpeptidase levels rose over the next three months to $334 \mathrm{IU} / 1$ and $106 \mathrm{IU} / 1$ respectively. Radioisotope liver scan showed multiple filling defects consistent with tumour deposits. Ascites and ankle oedema developed, and he died suddenly in January 1980 from an intraperitoneal bleed after a liver biopsy. Necropsy showed that a large haemorrhagic tumour, occupying most of the right lobe of the liver, had ruptured into the peritoneum. Several smaller haemorrhagic tumours were found elsewhere in the liver. An enlarged spleen ( $w t 760 \mathrm{~g}$ ) and thrombosed oesphageal varices were also noted. Histology showed the tumours to be angiosarcomas, with areas of sinusoidal dilatation and portal fibrosis in unaffected areas of the liver.

\section{Discussion}

Vinyl chloride monomer is transformed by hepatic microsomal enzymes to toxic metabolites that covalently bind to DNA.? After exposure to VCM hepatocytic proliferation, sinusoidal lining cell proliferation, and focal sinusoidal dilatation occur ${ }^{5}$ and angiosarcoma may later develop from the sinusoidal lining cells. Enlarged lipocytes may be seen in the space of Disse ${ }^{8}$; these cells are fibroblast precursors and can lay down collagen. ${ }^{9}$ Hepatic fibrosis results and may be associated with presinusoidal portal hypertension. ${ }^{10}$ It is commoner than angiosarcoma. ${ }^{2}$ Although fibrosis was found in tumour-free portions of the liver tissue from VCM workers dying of angiosarcoma, ${ }^{3}$ transition of hepatic fibrosis to angiosarcoma, although postulated, ${ }^{3}$ has not been observed.

The two patients described here were originally included in a series of seven VCM workers with portal hypertension. ${ }^{2}$ They were followed for five and 10 years respectively from the time of diagnosis of portal hypertension to death from angiosarcoma. During this period they were not further exposed to VCM. Their case histories indicate that hepatic fibrosis in a VCM worker may be a precursor of future malignant change and life-long follow-up is necessary. We know of only one other similar patient who died seven years after a portacaval shunt from an angiosarcoma. ${ }^{6}$

Non-cirrhotic fibrosis can also occur in the absence of portal hypertension, and may then be difficult to detect. It does not lead to a disturbance of liver function tests and may even be missed on needle biopsy of the liver. ${ }^{2}$ Greyscale ultrasonography is a useful diagnostic aid, ${ }^{11}$ but it has yet to be used in a large industrial survey, and there are probably several unrecognised affected VCM workers. Stringent measures to control levels of exposure should lead to eventual disappearance of noncirrhotic fibrosis. Those patients who already have the condition, however, are still at risk of developing hepatic angiosarcoma in the future.

A problem highlighted by the second case is that angiosarcoma is, by definition, a vascular lesion, and there is a risk of uncontrolled haemorrhage after blind liver biopsy. We believe, therefore, that the diagnosis should be sought either by laparoscopic biopsy or by hepatic angiography.

We thank Dr D M D Evans and Professor Peter Scheuer for their help in interpreting the biopsy material.

\section{References}

${ }^{1}$ Creech JL, Johnson MN. Angiosarcoma of the liver in the manufacture of polyvinyl chloride. JOM 1974;16:150-1.

${ }^{2}$ Smith PM, Crossley IR, Williams DMJ. Portal hypertension in vinyl chloride monomer workers. Lancet 1976;ii:602-4.

${ }^{3}$ Popper H, Maltoni C, Selikoff IJ. Vinyl chloride-induced hepatic lesions in man and rodents. A comparison. Liver 1981;1:7-20.

4 Tamburro CH. The hepatic role in carcinogenesis and its early detection-the vinyl chloride model. Yale J Biol Med 1978;51:67-80.

5 Popper H, Thomas LB, Telles NC, Falk H, Selikoff IJ. Development of hepatic angiosarcoma in man induced by vinyl chloride, thorotrast and arsenic. Am J Pathol 1978;92:349-69.

- Makk L, Delmore F, Creech JL, et al. Clinical and morphological patterns of hepatic angiosarcoma in vinyl chloride workers. Cancer 1976;37:149-63.

${ }^{7}$ Ostermann-Golkar S, Hultmark D, Segerback D, et al. Alkylation of DNA and protein in mice exposed to vinyl chloride. Biochem Biophys Res Commun 1977;76:259-66.

- Schaffner F, Popper H, Selikoff IJ. Initial features of vinyl chloride (VC) hepatic injury. Gastroenterology 1976;72:A35.

' Kent G, Gay S, Inouye T, Bahu R, Minick OT, Popper H. Vitamin A containing lipocytes and formation of type III collagen in liver injury. Proc Natl Acad Sci USA 1976;73:3719-22.

${ }^{10}$ Blendis LM, Smith PM, Laurie BW, Stephens MR, Evans WD. Portal hypertension in vinyl chloride monomer workers. A hemodynamic study. Gastroenterology 1978;75:206-11.

" Williams DMJ, Smith PM, Taylor KJW, Crossley IR, Duck BW. Monitoring liver disorders in vinyl chloride monomer workers using greyscale ultrasonography. Br J Ind Med 1976;33:1527. 